\title{
Percepción de competencia del profesorado de educaciónfísica con experiencia sobre la tarea como recurso didáctico \\ Perceived competence of experienced physical education teachers about the task as a didactic resource
}

\author{
Mario Díaz del Cueto, Raquel Aguado Gómez \\ Universidad Autónoma de Madrid
}

\begin{abstract}
Resumen: El estudio que se presenta en el presente artículo trata de conocer la percepción del profesorado sobre su propia competencia referida al conocimiento del contenido y a la tarea como herramienta didáctica. En el estudio participaron un total de 173 docentes en Educación Física que realizan su función en las etapas de Primaria y Secundaria en 9 Comunidades Autónomas. Para la obtención de información cumplimentaron un cuestionario, realizándose, asimismo, un análisis audiovisual de 10 clases previamente grabadas. Los resultados muestran que los docentes, independientemente de la etapa educativa en la que imparten su docencia, de los años de experiencia docente y del tipo de tarea, buscan que el alumnado participe (con un sentido más lúdico o deportivo según la etapa educativa) de forma organizada y manteniendo las normas establecidas, no observándose modificaciones o ajustes de los modelos previamente verbalizados y/o ejemplificados.

Palabras clave; Autopercepción; competencias docentes; contenidos; tareas; Educación Física.
\end{abstract}

Abstract: The study presented in this article attempts to comprehend the perception of teachers about their own competence in terms of knowledge of content and tasks as a teaching tool. The study included 173 physical education teachers while performing their role in Primary and Secondary stages in 9 autonomous regions. Information was obtained from a questionnaire, and analysis was carried out on 10 previously recorded classes. The results show that teachers, independently of the educational stage which is taught, years of teaching experience and type of task that students seeking to participate (with a more playful or sport as an educational stage) of maintaining an organized and established standards, observed no changes or adjustments to the models previously verbalized and / or exemplified.

Key words: Self-perception; teaching skills; content; tasks; Physical Education.

\section{Introducción}

El interés sobre la eficacia del profesorado se ha incrementado de forma considerable en las últimas décadas. Este aspecto del comportamiento deldocente es considerado comoun elemento multidimensional y específico de cada contexto de intervención (Skaalvik \& Skaalvik, 2007). En este sentido, Bandura define la auto eficacia percibida como «los juicios que realizan las personas sobre sus capacidades para organizar y llevar a cabo acciones encaminadas a alcanzar determinados resultados» (Bandura, 1986, p.391).

Atendiendo al contexto escolar, la percepción de autoeficacia docente puede ser definida como «as creencias individuales que tiene un profesor sobre sus capacidades para desarrollar tareas específicas de enseñanza con un nivel específico de calidad en una situación específica» (Dellinger, Bobbett, Oliver \& Ellett, 2008, p.752). Por lo tanto la concepción que se tenga sobre la calidad de la enseñanza va a ser determinante, así como la especificidad del contexto.

La función que deben asumir los docentes al impartir su materia debería estar orientada hacia la observación de las situaciones que se generan en el proceso de enseñanza y aprendizaje. En este sentido, se les demanda la capacidad para reflexionar y tomar decisiones sobre la base de la inestabilidad de las situaciones en donde acontece su labor profesional (Schön, 1998; Tinnig, 2002).

Esta forma de entender la enseñanza conlleva la posibilidad de «emitir criterios sobre la práctica en un contexto de significados y valores compartidos» (Ingvarson \& Kleinhenz, 2006, p. 265). Friedman y Kass (2002) definieron la autoeficacia docente como «a percepción de la habilidad del profesorado para: a) realizar las tareas profesionales requeridas y para regular las situaciones implicadas en el proceso de enseñanza y aprendizaje de los estudiantes (eficacia de la clase) y b) realizar tareas organizativas, siendo parte de la organización y de sus procesos políticos y sociales (eficacia organizativa)» (p. 684).

El interés por determinar cuáles son las competencias que debe tener un docente para que se considere de calidad, ha preocupado a profesionales e instituciones. Un ejemplo pueden ser las establecidas

Fecha recepción: 14-01-12 - Fecha envío revisores: 14-01-12 - Fecha de aceptación: 04-02-12 Correspondencia: Mario Díaz del Cueto

Av. Tomás y Valiente. Campus de Cantoblanco.

Av. Tomás y Valiente. Campus de Cantoblanco.
Ctra. Colmenar Viejo, Km. 15,500. 28049 Madrid. )

E-mail: mario.diaz@uam.es por la National Board for Professional Teaching Standards (NBPTS), en el presente artículo hacemos referencia a las que están más directamente relacionadas con el objeto de estudio de este documento, en donde: a) los profesores están comprometidos con los estudiantes y con su aprendizaje, b) los profesores saben los temas que enseñan y cómo enseñarlos a los estudiantes y c) los profesores son responsables de orientar y de supervisar el aprendizaje del estudiante (NBPTS, 2006).

Posiblemente una de las cuestiones que en las últimas décadas ha despertado el interés por estos estudios, han sido algunos de los resultados obtenidos relacionados con las creencias que tiene el profesorado sobre sus capacidades de enseñanza y la importante influencia que tienen sobre la efectividad de su enseñanza (Tschannen-Moran \& Woolfolk Hoy, 2007; Knoblauch \& Hoy, 2008).

La percepción de autoeficacia docente es algo dinámico, que cambia en función del contexto, de las experiencias previas, de la formación, así como de factores afectivos (Fores Ramírez, 2001; Friedman \& Kass, 2002, Knoblauch \& Hoy, 2008) encontrándose periodos estabilidad y otros de cambio en distintos periodos de la experiencia docente (Knoblauch \& Hoy, 2008).

Las experiencias previas son determinantes en la percepción de autoeficacia (Bandura, 1986; Hoy \& Spero, 2005; Knoblauch \& Hoy, 2008) por lo tanto los años de experiencia docente juegan un papel determinante, pudiéndose encontrar diferencias entre profesorado con pocos años de experiencia y profesorado más experimentado. Diversos estudios han señalado que la percepción de autoeficacia en profesores novatos se ve más influenciada por factores contextuales que los profesores más experimentados (Tschannen-Moran \& Woolfolk Hoy, 2007)

El estudio realizado tuvo como objetivo: conocer la percepción del profesorado sobre su propia competencia en relación con el conocimiento del contenido y la utilización de tareas como herramienta didáctica

\section{Método}

\subsection{Participantes}

En el estudio han participado 173 docentes (111 profesores y 62 profesoras) con experiencia en Educación Física y que hemos distribuido en bloques de 5 años correspondientes con: $<5 ; 5-10 ; 11-15$ y $>16$ 
respectivamente. Estos docentes pertenecían a 9 Comunidades Autónomas y realizaban su función profesional en las etapas educativas de Primaria y Secundaria.

\subsection{Instrumento y técnicas de recogida de datos}

Cuestionario; de acuerdo con las aportaciones de la revisión bibliográfica realizada y atendiendo a las necesidades del estudio, se diseñó un cuestionario al efecto, con una escala de valoración de 0 a 5 , representando el 0 no estar nada de acuerdo y 5 estar totalmente de acuerdo. Este cuestionario recoge las competencias docentes en cinco dimensiones y en este artículo hacemos referencia a dos de ellas, a la percepción que tiene el profesorado sobre su Conocimiento del contenido de enseñanza y sobre su Conocimiento didáctico del contenido, centrándolo en la segunda dimensión para este artículo en la sub-dimensión Actividades y tareas.

Grabación de clases en vídeo y audio; con la intención de darle sentido a los datos recogidos a través del cuestionario, hemos realizado un análisis de las clases grabadas a 10 de estos profesores y profesoras. El propósito de las grabaciones ha sido conocer la aplicación didáctica de las tareas como herramienta del aprendizaje.

Tanto en la aplicación del cuestionario como en el análisis de los vídeos se ha asegurado el anonimato de los participantes.

\subsection{Tratamiento de los datos}

Cuestionario: se realizó un análisis estadístico atendiendo a la media y deviación típica y utilizando el programa SPSS versión 17.0 (SPSS Inc., Chicago, Illinois, USA).

Grabación en audio y vídeo: se realizó un análisis interpretando la intervención docente, en función de la dimensión y sub-dimensión anteriormente mencionadas y observables durante el proceso de enseñanza y aprendizaje.

Tanto para la aplicación del cuestionario comô para el análisis de los videos, se ha asegurado plenamente el anonimato de los participantes y utilizando nombres ficticios en el caso de los vídeos.

\section{Resultados y discusión}

Como primera información señalaremos que no se han encontrado diferencias significativas relacionadas con: la variable género, etapas educativas de Primaria y Secundaria, ni entre ninguna de las dimensiones analizadas. Se ha podido observar que la percepción de competencia del profesorado encuestado, se manifiesta en todos los ítems por encima del valor considerado de grado medio (3).

\section{Percepcción sobre el contenido, materia y docencia}

Todos los promedios, independientemente de la competencia a la que haga alusión o de los años de experiencia, se encuentran enmarcados dentro de los valores que consideramos medio-altos.

Los promedios totales del profesorado nos muestran que se perciben con un alto nivel de competencia sobre el dominio de la asignatura a modo general lo que les hace sentirse seguros y tranquilos al comenzar

\begin{tabular}{|c|c|c|c|c|c|c|c|c|c|c|}
\hline & & & & \multicolumn{7}{|c|}{ Años de experiencia doce nte } \\
\hline & \multicolumn{4}{|c|}{$<5$} & \multicolumn{2}{|c|}{$5-10$} & \multicolumn{2}{|c|}{$11-15$} & \multicolumn{2}{|l|}{$>16$} \\
\hline & M & DT & M & DT & M & DT & M & DT & M & DT \\
\hline $\begin{array}{l}\text { Puedo a frontar con compe encia el de sarrolb de las } \\
\text { clases seacual se a e l contenido que vaya a utilizar }\end{array}$ & 3.32 & 973 & 3.11 & 1.09 & 3.49 & .88 & 3.37 & 94 & 3.27 & .99 \\
\hline $\begin{array}{l}\text { Estoy con vencido de que puedo empezar las clases con } \\
\text { tranquilidad, ya que eldom inio de la asgna tura me } \\
\text { aport a suficiente seguridad. }\end{array}$ & 4.297 & .793 & 3.98 & .91 & 4.31 & .87 & 4.55 & 61 & 4.28 & 68 \\
\hline $\begin{array}{l}\text { Estoy plenamente conve ncido de mis capacidades } \\
\text { docentes oomo profesora o profesor de Educación Fís ca. }\end{array}$ & 4.13 & .854 & 4.17 & .87 & & .92 & 4.24 & .62 & 3.99 & .93 \\
\hline \multicolumn{11}{|c|}{ Tabla 2. Percepción sobrel ata rea como herramienta didáctic a según años de ex periencia } \\
\hline & & & \multicolumn{8}{|c|}{ Años de experie ncia docente } \\
\hline & & & \multicolumn{2}{|c|}{$<5$} & $5-10$ & & \multicolumn{2}{|c|}{$11-15$} & \multicolumn{2}{|c|}{$>16$} \\
\hline & M & DT & M & DT & $\mathrm{M}$ & DT & M & DT & M & DT \\
\hline $\begin{array}{l}\text { Siento que soy un docente hábil para modi ficar sobre la } \\
\text { marcha unat rea y ad aptarla así a las circunstancias } \\
\text { concretas del momento. }\end{array}$ & 4.16 & .85 & 3.90 & .92 & 4.25 & .86 & 4.27 & .75 & 4.18 & .84 \\
\hline $\begin{array}{l}\text { Estoy capacitado parapresentar con claridad y precisión las } \\
\text { diversas tare ax de enseñanza. }\end{array}$ & las 3.89 & .81 & 3.83 & .76 & 3.81 & .92 & 3.96 & .76 & 3.96 & .80 \\
\hline $\begin{array}{l}\text { Soy capaz de explicar a las alumnas y alumnos bs motivos } \\
\text { por los que realiz an una tare ade forma incorrecta. }\end{array}$ & 4.00 & .81 & 3.83 & .88 & 4.06 & .85 & 3.96 & .82 & 4.10 & .72 \\
\hline
\end{tabular}

las clases. Su percepción disminuye ligeramente al hacer referencia a sus capacides como docentes, reduciéndose hasta valores medios cuando se les pregunta por su dominio de los contenidos.

El profesorado que se percibe con mayor nivel de competencia sobre el dominio de la asignatura es el que está dentro del bloque de 11 a 15 años de experiencia, siendo el nivel más bajo en esta competencia el manifiestado por el profesorado con menor experiencia ( $<5$ años).

Con cierta sorpresa, observamos que es el profesorado con más experiencia (>16 años) el que manifiesta percibirse con menor nivel de competencia como docente.

Cuando se concreta la competencia docente haciendo alusión al dominio de los contenidos, los docentes que se manifiestan con mayor competencia, aunque dentro de un nivel medio, son los que tienen un margen de experiencia docente entre 5-10 años. Contrasta este nivel de percepción con los valores más bajos que que presentan otros profesores y profesoras con más años de experiencia docente.

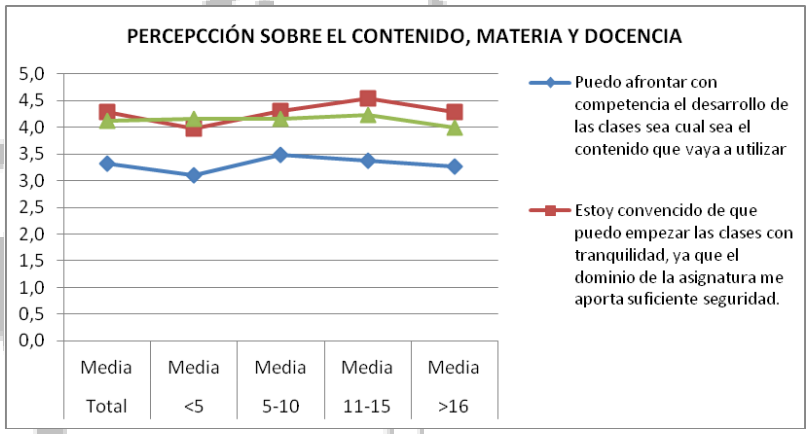

Figura 1. Percepción sobre el contenido, materia y docencia

Percepción de competencia sobre la tarea como herramienta didáctica

Los promedios totales muestran mayor percepción de competencia del profesorado en su habilidad para modificar las tareas durante la interacción con el alumnado y adaptándola a las características que observa durante el desarrollo de las clases; así como en la que hace referencia a explicar al alumnado los motivos de una inadecuada realización.

En el análisis de los vídeos, no se ha observado ninguna modificación en relación a la tarea inicial para ajustarla a los distintos niveles de realización que muestra el alumnado en ninguno de los segmentos de experiencia docente. El profesorado comienza la sesión presentando la tarea que ha diseñado y todo el alumnado la realiza según las pautas iniciales. Bien sea con una demostración gestual/descripción verbal del gesto técnico a realizar (p.e. pase de balón; lanzamiento;...) o con una situación problema (p.e. 2 vs2; realizar una figura entre varios alumnos y alumnas).

Asimismo, todo el alumnado, independientemente de su nivel de destreza, realiza la misma tarea y con las mismas condiciones que inicialmente estableció el docente. Esta cuestión se aleja de las aportaciones que se reflejaban de forma relevante en la actuación docente y que hacían referencia a la capacidad para reflexionar y tomar decisiones sobre la base de la inestabilidad de las situaciones la labor profesional (Schön, 1998; Tinnig, 2002), pero que coincide con los resultados obtenidos por Velázquez et al (2007) en los que señalan que prácticamente no existen adaptaciones de las tareas en función de la diversidad de capacidades motrices.

En este sentido, se observa de forma reiterativa una asunción por parte de los docentes de un rol centrado en el aporte de información normativa, como podemos observar en la manifestación de Antonio:

«Intentemos que no quede ninguna pelota en nuestro campo» (Antonio/>16)/Pri/JPopulares)

«Así no, lo cojo con las dos manos» (Sara/entre 5-10/Pri/ HEspecíficas)

Otra de las actuaciones docentes que se observa de forma continuada tienen relación con el aporte de información de tipo motivacional. Se puede observar en las siguientes manifestaciones: 

tivos)

«Bien, y hay que concentrarse» (Roberto/(entre 5-10/Pri/Alterna-

«Muy bien Pepe» (Iván/entre 10-15/Sec/ Bádminton)

Este análisis ha permitido observar que el profesorado no realiza correcciones o ajustes que orienten al alumnado sobre otras posibilidades de realización. En este sentido, consideramos que puede estar motivado por la influencia que todavía existe desde la enseñanza tradicional en donce larigidez de la planificación, el control de la clase y la reprodución de las tareas previamente establecidas con una rigidez manifiesta (Romero Cerezo, 2004), puede ser uno de los motivos que provoque dichos comportamientos docentes.

Posiblemente, la no adaptación del grado de dificultad de las tareas en un sentido amplio a la diversidad del alumnado, sea uno de los motivos por los cuales las competencias que hacen referencia a su percepción sobre la motivación del alumnado desde la tarea y, sobre todo, que el alumnado comprenda las habilidades implicadas en las tareas, muestren los promedios más bajos en la percepción de competencia de los docentes e independientemente del segmento de años de experiencia, mostrando esta última competencia los mismos promedios en los segmentos $<5 \mathrm{y}>15$.

Por lo observado de forma audiovisual, los docentes, independientemente de la etapa educativa, años de experiencia docente y tipo de tarea, buscan que el alumnado participe -con un sentido más lúdico o deportivo según la etapa educativa- durante la realización de las tareas de forma organizada y manteniendo las normas de disciplina establecidas,

Además, no se han observado correcciones o ajustes de los modelos técnicos previamente verbalizados y/o ejemplificados.

Se ha podido observar en el análisis de los videos que, independientemente de los años de experiencia y del contenido a utilizar, el profesorado utiliza principalmente tareas que participan de dos situaciones: analíticas mostrando los contenidos de forma secuencializada y situaciones globales acercándose a los contextos del juego real, con una aproximación a las que Famose (1992) define como tareas semidefinidas.

\section{Conclusiones}

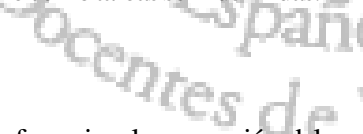

Como principales conclusiones y en referencia a la percepción del profesorado sobre su competencia docente referida al contenido, materia, docencia y tarea, consideramos que:

- El profesorado que ha participado en este estudio, se muestra con una percepción media-alta de su competencia sobre el contenido, la materia y la docencia. No encontrándose diferencias significativas en función de la variable género, ni de la etapa educativa en la que el profesorado imparte su docencia.

- Respecto a la percepción de su competencia sobre la tarea como herramienta didáctica, en los aspectos en los que su percepción de competencia es mayor es en su habilidad para modificar tareas durante la interacción con el alumnado, así como la adaptación de las tareas en aspectos que observa durante el desarrollo de las sesiones de clase.

Esta última percepción se contrapone a los resultados del análisis realizado a través de los videos de las sesiones de clase, ya que durante su implementación el profesorado no realiza correcciones que orienten al alumnado sobre otras posibilidades de realización y no se produce ninguna modificación delas tareas atendiendo a ladiversidad delalumnado.

\section{Referencias}

Bandura, A. (1986). Social foundations of thought and action: A social cognitive theory. Englewood Cliffs, NJ: Prentice-Hall.

Dellinger, A. B., Bobbett, J. J., Olivier, D. F., \& Ellett, Ch. D. (2008). Measuring teathers' self-efficacy beliefs: Development and use of the TEBS-Self. Teaching and Teacher Education, 24(3), 751-766.

Famose, J. P. (1992). Aprendizaje motor y dificultad de la tarea. Barcelona: Paidotribo.

Flores Ramírez, M. D. (2001). El factor humano en la docencia de educación secundaria: un estudio de la eficacia docente y el estrés a lo largo de la carrera profesional. Tesis doctoral, Universidad Autónoma de Barcelona. Recuperado de http://www.tesisenxarxa.net/ TDX-1004102-142853/

Friedman, I. A., \& Kass, E. (2002). Teacher self-efficacy: A classroomorganization conceptualization. Teaching and Teacher Education, 18(6), 675-686.

Knoblauch, D., \& Hoy, A. W. (2008). «Maybe I can teach those kids.» The influence of contextual factors on student teachers' efficacy beliefs. Teaching and Teacher Education, 24(1), 166-179.

Hoy, A. W., \& Spero, R. B. (2005). Changes in teacher efficacy during the early years of teaching:Acomparison of four measures. Teaching and Teacher Education, 21(4), 343-356.

Ingvarson, L. \& Kleinhenz, E. (2006). Estándares profesionales para la práctica y su importancia para la enseñanza. Revista de educación, 340, 265-295.

NATIONAL BOARD FOR PROFESSIONAL TEACHING STANDARD (2006). What Teachers Should Know and Be Able to Do. Recuperado de www.nbpts.org/resources/publications

Romero Cerezo, C. (2004). Argumentos sobre la formación inicial de los docentes en educación física. Profesorado, revista de currículum y formación del profesorado, 8(1). Recuperadode www.ugr.es/ $\sim$ recfpro/rev81ART5.pdf

Schön, D. A. (1998). El profesional reflexivo. Cómo piensan los profe- sionales cuando actúan. Barcelona: Paidós.

Skaalvik, E. M., \& Skaalvik, S. (2007). Dimensions of teacher selfefficacy and relations with strain factors, perceived college teacher efficacy, and teacher burnout. Journal of Educational Psychology, 99(3), 611-625.

Tinnig, R. (2002). Engaging Siedentopian Perspectives on Content Kowledge for Physical Education. Journal of Teaching in Physical Education, 21,368-377.

Tschannen-Moran, M., \& Woolfolk Hoy, A. (2007). The differencial antecedents of self-efficacy beliefs of novice and experienced teachers. Teaching and Teacher Education, 23(6), 944-956.

Velázquez Buendía, R., Hernández Álvarez, J. L., Garoz Puerta, I., López Crespo, C., López Rodríguez, Á., Maldonado Rico, A.... Castejón Oliva, F. J. (2007). Calidad de enseñanza en Educación Física y Deportiva y discurso docente: El caso de la Comunidad de Madrid. Revista de Educación, 344, 447-467.

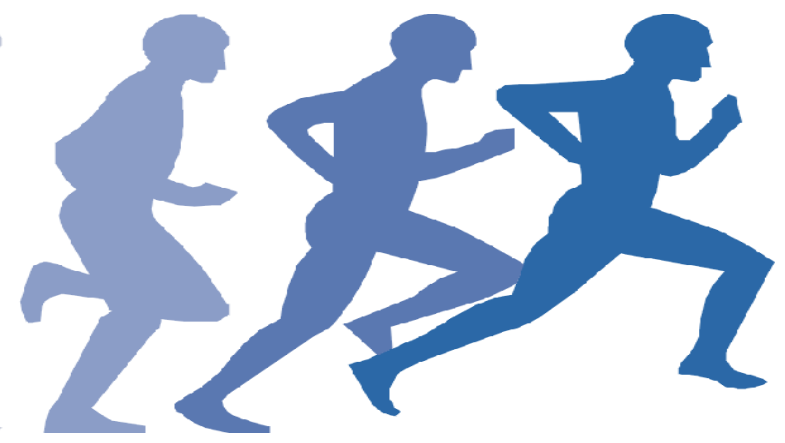

